\title{
The Preparation of Nano Silver by Chemical Reduction Method
}

\author{
Tevfik Raci Sertbakan', Emad K. Al-Shakarchi' ${ }^{2}$, Saif Sultan Mala ${ }^{1}$ \\ ${ }^{1}$ Kırşehir Ahi Evran University, Art and Science Faculty, Department of Physics, Kurşehir, Turkey \\ ${ }^{2}$ Al-Nahrain University, College of Science, Physics Department, Baghdad, Iraq \\ Email: trsertbakan@ahievran.edu.tr,eks2000@hotmail.com
}

How to cite this paper: Sertbakan, T.R. Al-Shakarchi, E.K. and Mala, S.S. (2022) The Preparation of Nano Silver by Chemical Reduction Method. Journal of Modern Physics, 13, 81-88. https://doi.org/10.4236/jmp.2022.131006

Received: December 8, 2021

Accepted: January 25, 2022

Published: January 28, 2022

Copyright (c) 2022 by author(s) and Scientific Research Publishing Inc. This work is licensed under the Creative Commons Attribution International License (CC BY 4.0).

http://creativecommons.org/licenses/by/4.0/

\section{(c) (i) Open Access}

\begin{abstract}
A silver nanostructures prepared by using chemical reduction method. The silver nanoparticles were prepared with diameters of about $(20 \mathrm{~nm})$. Numerous techniques had been used to study the optical, structural like the UV-Vis absorption spectrometer, Ttransmission Electron Microscopy (TEM), Field-Emission Scanning Electron microscope (FESEM), and X-ray diffraction (XRD). The practical results exhibited the absorption spectrum of the prepared nanoparticles at $(357 \mathrm{~nm})$, it was found that there is a relationship between the positions of the optical absorption peak and the size of the silver nanoparticles. The analysis of TEM results showed the presence of nanoparticles in the range $(20$ $\mathrm{nm}$ ). The analyzing of XRD results explained the crystal structure for silver nanoparticles. It is found a cubic unit cell have a lattice constants $(\mathrm{a}=4.0855$ $\AA$ ), with the Miller indices were (111), (002), (002), and (113).
\end{abstract}

\section{Keywords}

Chemical Reduction Method, UV-Vis Absorption Spectrometer, Field-Emission Scanning Electron Microscope, Ttransmission Electron Microscopy

\section{Introduction}

The nano materials are rapidly developing recently through potential applications in a wide range of technological areas such as electronics, catalysis, ceramics, magnetic data storage, structural components [1]. Silver nanoparticles (AgNPs) are very important among different metallic nanoparticles, because it had a biomedical applications especially in cancer diagnosis and therapy. A nano spherical silver powder prepared using a chemical reduction method. The Turkevich procedure [2] used for synthesis AuNPs, it considered most probable many reasons. The procedure is very simple and reliably to produces AuNPs 
with diameters range $(5-150 \mathrm{~nm})$. The second thing, it is nontoxic and stabilized by citrate ions [3]. There is a fast nucleation in the synthesis leads to AuNPs with narrow size distribution [3] [4]. It was achieved by increasing the reactivity of the precursor. There are two procedure used to generate nanoparticles depend on a limited amounts of a material provided. Commonly, they applied in most researches and studies to produce nanostructure materials. These are classified as, the bottom-up and the top-down techniques, both mentioned in Figure 1. The bottom-up is assembled atoms together to produce nanoparticle or dis-assemble the bulk material producing nanostructure, the last one represent the up-down technique. The first procedure is more complicated because it needs high technology to assemble many atoms or molecule producing nanostructure. The second is simpler and it needs a simple equipment to perform laboratory because it depends on the decomposition of bulk material into nanostructure. There many chemical and physical process used to transfer the bulk material into nanostructure such as nanoparticles, but the reduction method is one of powerful technique used to perform nanoparticle in the procedure top-down [5] [6].

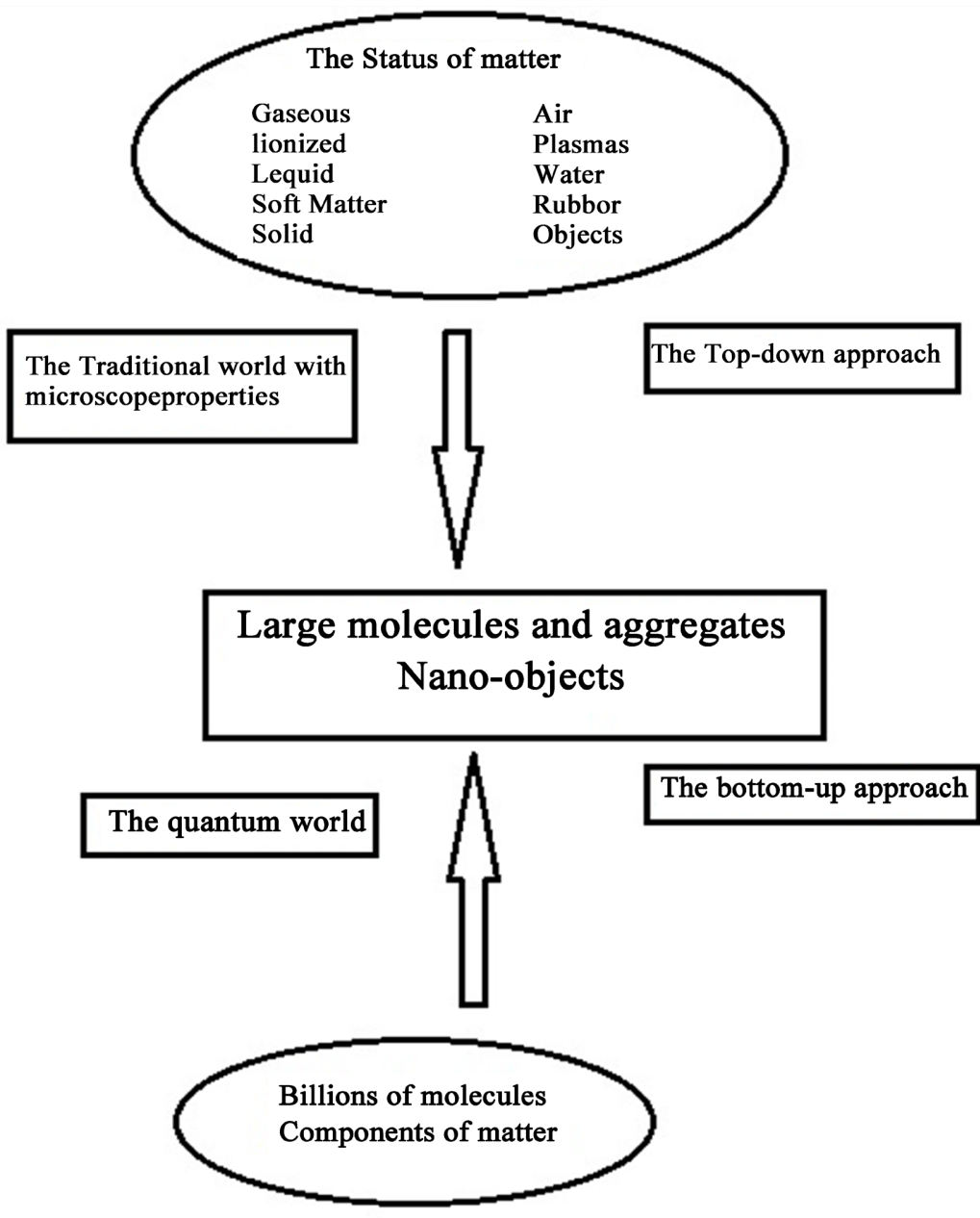

Figure 1. The technique of nanostructure [7]. 
In this research, it is related to prepare a silver nanoparticle experimentally by using the reduction method. It is necessary to apply in the laboratory and making a specification on the sliver nanoparticle represented by Transmission Electron Microscope (TEM), Field-Emission Scanning Electron microscope (FESEM), UV-VIS spectrophotometer, and X-ray diffraction. All these analysis give us a knowledge on the characterization Ag-nanoparticle prepared experimentally.

\section{Experimental Procedure}

The reduction method is one of the chemical methods those were used to prepare silver nanoparticles. It is considering a bottom-up procedure because the chemical reduction tends to produce Ag-atoms which are assembled to generate nanostructures. During the synthesis process, if the reaction rate is too fast, rapid formation of a large amount of metal nuclei will occur and result in particles that are too small. On the other hand, agglomeration of particles will take place if the reaction rate is too slow. The principle chemicals used in the experiment were the silver nitrate $\left(\mathrm{AgNO}_{3}\right)$, glucose and polyvinyl pyrrolidone (PVP), and sodium hydroxide. The Silver nanoparticles reduced from the silver nitrate in PVP aqueous solution. The glucose used as reducer and sodium hydroxide apply to accelerate the reaction. Firstly, the preparation of silver nitrate solution prepared by adding $3.4 \mathrm{~g}$ of $\mathrm{AgNO}_{3}$ into $20 \mathrm{ml}$ distilled water, the solution (a). The PVP solution prepared by dissolving PVP, the glucose and sodium hydroxide in $60 \mathrm{ml}$ distilled water together, producing solution (b). Then the solution (b) heated to temperature $\left(60^{\circ} \mathrm{C}\right)$ by magnetic stirrer. After the temperature fixed for $(15 \mathrm{~min})$, the solution (a) added to (b) by drops. Then the mixed solution was stirred for $(10-15 \mathrm{~min})$. The particles were separated by centrifugation, and washing the products by distilled water many times to remove the possibility $\mathrm{NO}_{3}$ that might be not reacted [8]. There are many technique used to examine the appearance of nanoparticles. A field emission scanning electron microscopy (FESEM) and transmission electron microscope were used to investigate the nanostructure production. Normally, FESEM-device is giving a high resolution rather than SEM. That's making a fine grain in the nano size. The main difference between FESEM and TEM is creating image by detecting reflected electrons, while TEM uses transmitted electrons to create an image. Then TEM-results offers a valuable information on the inner structure of the sample, while SEM provides information on the surface morphology and its composition. X-ray diffraction technique (XRD) is using to define the structural nature of the prepared sample. To investigate this purpose, the application of CrysDiff software is necessary to make a simulation on the XRD-pattern.

\section{Results and Discussion}

After the successive preparation had been done by the reduction method to produce a silver nanoparticles. These analysis and measurements are useful to prove the successful the preparation of silver nanoparticle experimentally. The 
absorption spectrum of silver nanoparticle by UV-visible spectrophotometer. It had been done for all prepared samples as a function of different concentration. The analysis of absorption spectrum state the first step of understanding the excited electronic states of molecules. It gives more information from state $\left(\mathrm{S}_{\mathrm{o}}\right)$ to excited state $\left(\mathrm{S}_{\mathrm{p}}\right)$ for the molecules under investigation, as shown in Figure 1 \& Figure 2 for different concentration $1 \%$, and $2 \%$ respectively. The spectrum is depending on the concept of surface Plasmon of silver nanoparticles which had been prepared by chemical reduction method. The surface Plasmon spectrum of large silver nanospheres those were mentioned by the sample with concentration (1\%) as shown in Figure 2. It was clear that the maximum absorption peak occurred at $(357 \mathrm{~nm})$ with a peak intensity about (1.82 a.u). Whereas the surface Plasmon spectrum of silver nanostructure was denoted in Figure 3, for the concentration (2\%). One can noted the maximum absorption peak occurred at (358 $\mathrm{nm}$ ) with a peak intensity about (3.11 a.u).

It is clear that the maximum absorption peak of surface Plasmon absorbance of silver nanoparticles goes toward short wavelength. That is obvious the indication for the decreasing the size of nanostructure such as particles. These results

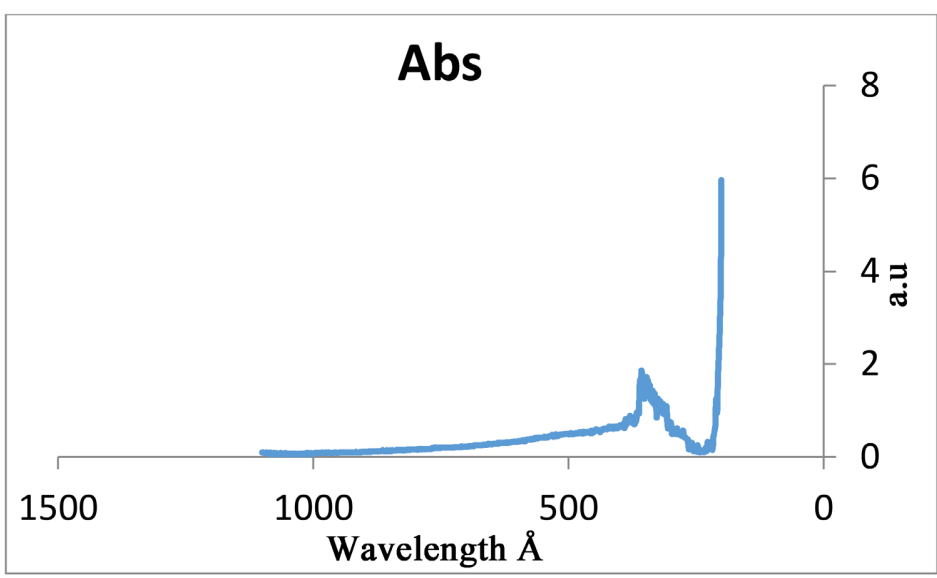

Figure 2. Indicate the UV-VIS spectrum for silver nanoparticle solution with $1 \%$ concentration.

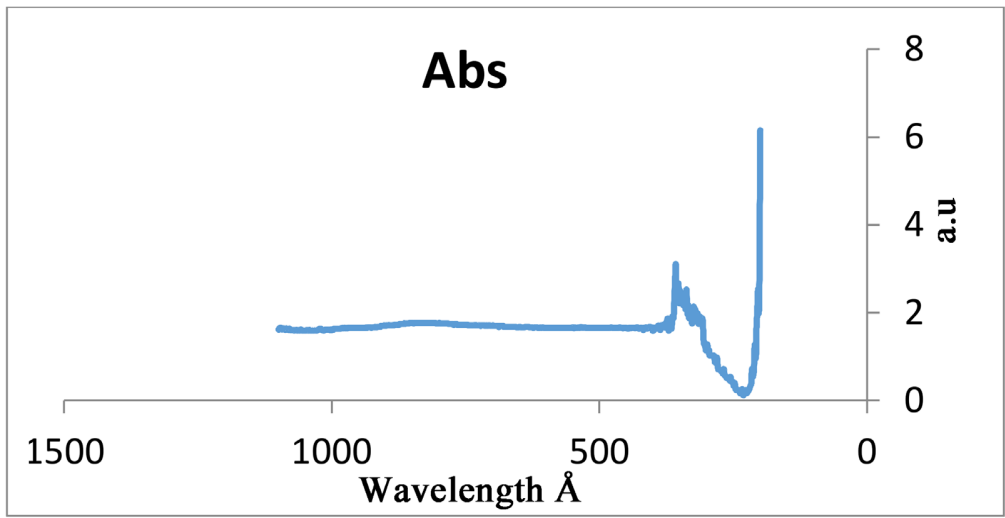

Figure 3. Indicate the UV-VIS spectrum for silver nanoparticle solution with $2 \%$ concentration. 
is confirmed with the results obtained from transmission electron microscope previously [9].

The transmission electron microscopy (TEM) is a good technique to observe the size and the shape of the silver nanostructures, as shown in Figure 4. It was appeared as spherical shape with average particle size in the range $(20 \mathrm{~nm})$. The silver nanoparticles also showed high monodispersed. The results of FESEM is compatible with TEM-results. It was cleared the presence the nanoparticle, in addition it gives the morphology of surface and the homogeneity of grain size and grain boundaries, appeared in Figure 5. The results showed the presence of silver nanoparticle in different size. In addition, the agglomeration of silver nanoparticle is clearer through the increasing of silver nanoparticle rather than the

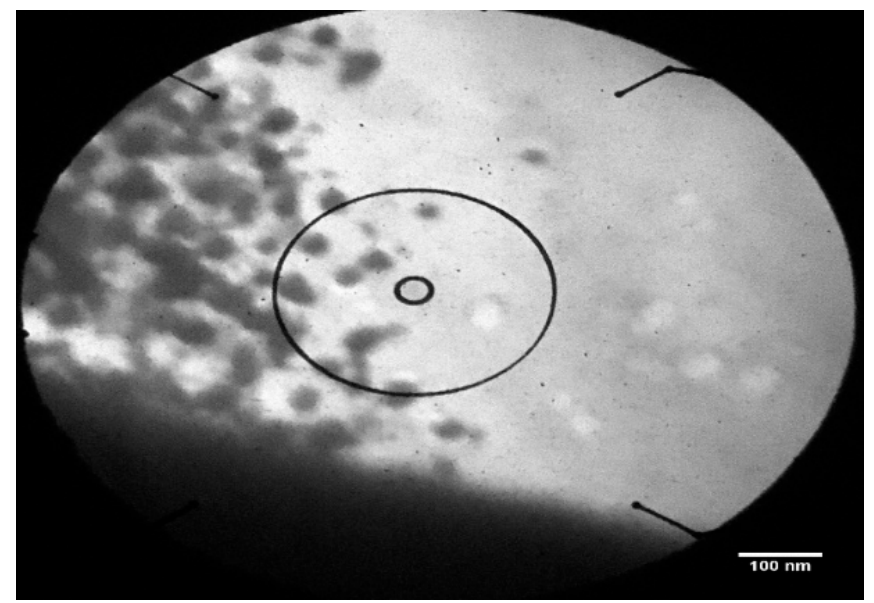

Figure 4. The TEM results for silver nanoparticle.

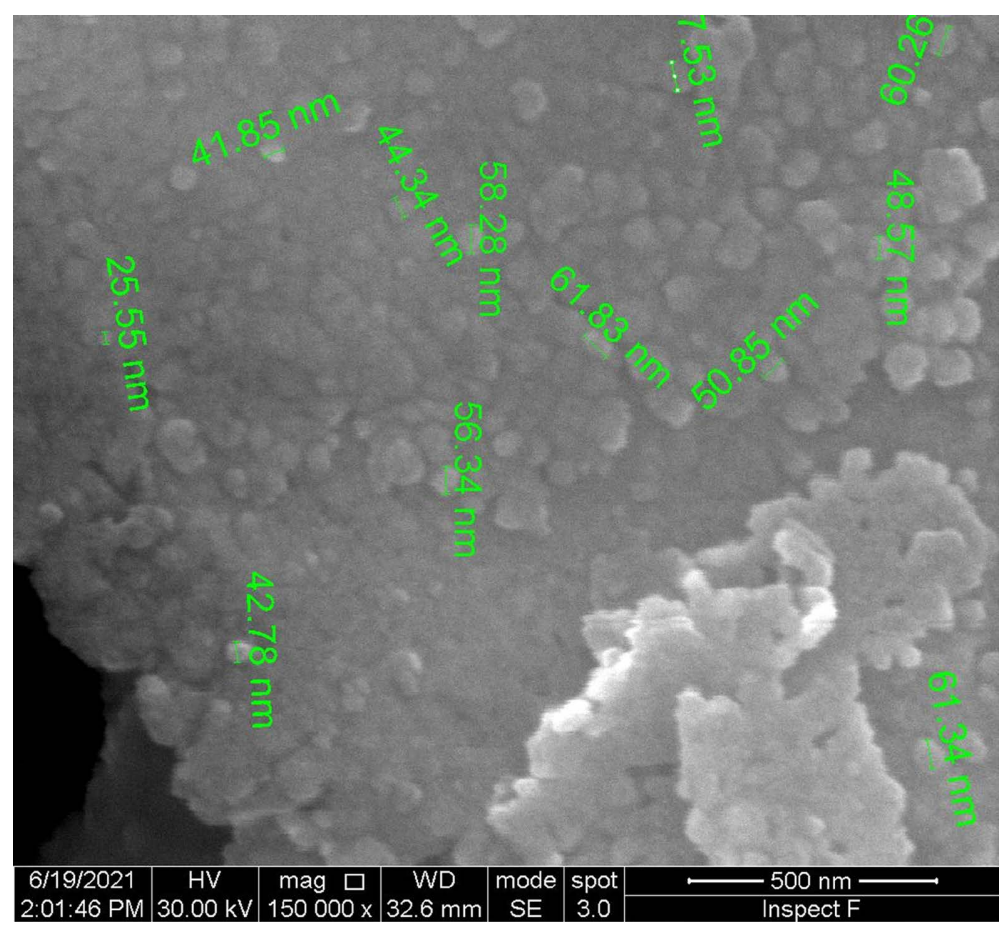

Figure 5. The FESEM results for silver nanoparticle with different position. 
size of nanoparticle concluded in TEM results. The agglomeration is possible because of high conductivity of silver metal under the effect of the electron beam of FESEM.

The crystal structure during the XRD-pattern, as shown in Figure 6. The pattern analyzed by using specialized software to find the type of structure and the related lattice parameters, through the simulation by CrystalDiff. Software. The simulated results comparing with related database is the standard chart $(1,100,136)$. The simulated data showed a cubic phase with lattice constants $(\mathrm{a}=\mathrm{b}=\mathrm{c}=4.0855 \AA$ ), and the volume of unit cell is $\left(68.1923 \AA^{3}\right)$. The peaks are mentioned by the miller planes labeled by (111), (002), (022), and (113). It is agreement with the Lai et al. [1] and in contradiction with Mehta et al. [10].

The determination of lattice constants, and the crystal phase is related to position of the peaks at a certain $(2 \theta)$, which are coincidence with the data base. The widen peaks in the X-ray pattern is considering the proof of nanoparticles appearance.

The appearance of many peaks is returning to polycrystalline structure, for this reason there are many orientation through the grains, and a predominant orientation is (111). Secondly, the peaks intensity is small enough comparable with X-ray diffraction of bulk sample. That is return the low density of Miller planes within the unit cell in comparable with bulk sample.

It is useful to determine the crystallite size inside the grains, and the elastic strain might be exhibited in the unit cell during the axis (a, b, c) by applying Williamson-Hall equation, as mentioned in Figure 7. The results showed the crystallite size is about (155.7808 $\AA$ ) which is equal to $(15.57 \mathrm{~nm})$. That is exactly equal to the grain size appeared. It is smaller than the particle size that was discussed by TEM-results before. The important thing that is mentioned related to

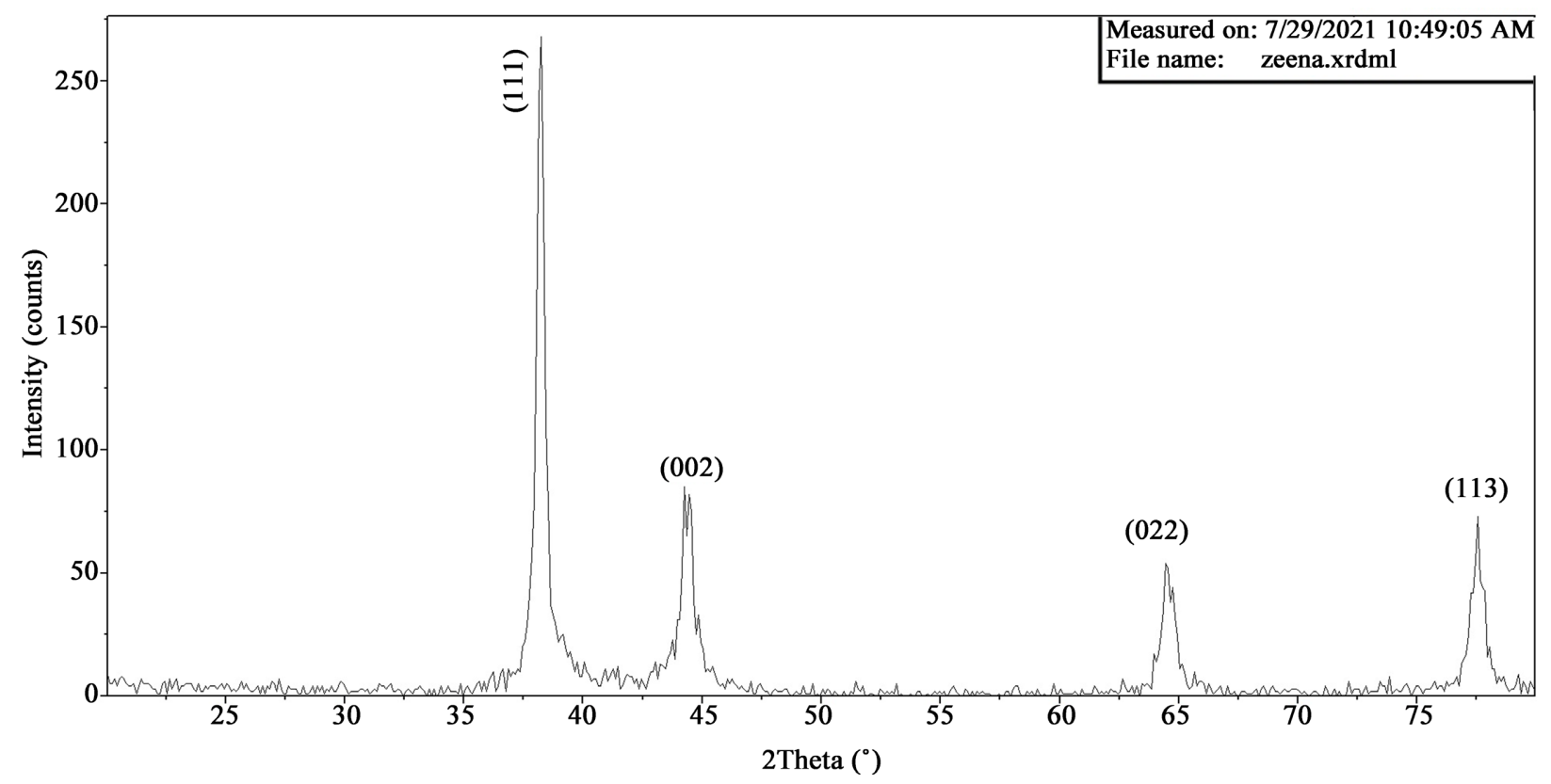

Figure 6. XRD pattern of silver nanoparticle. 


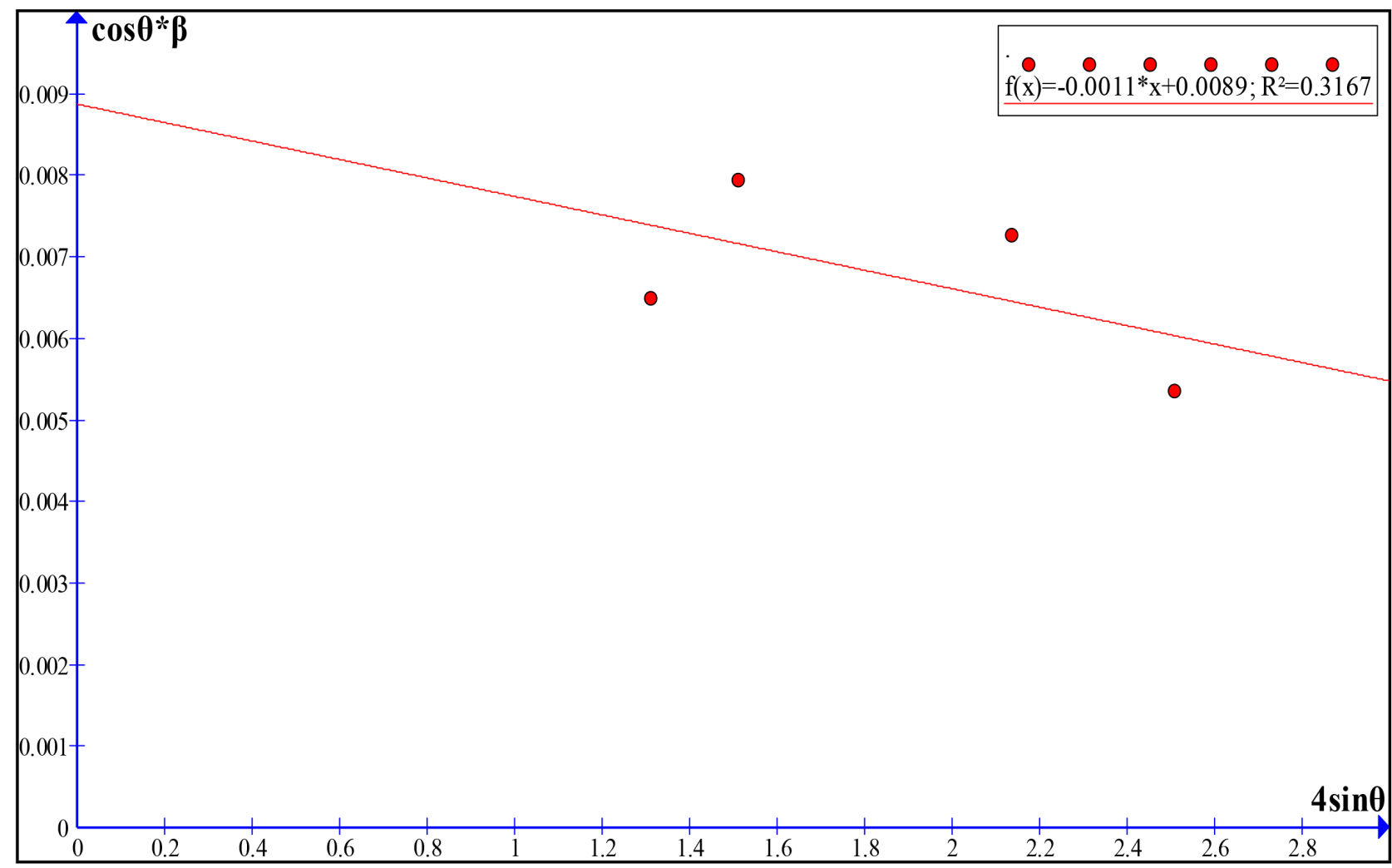

Figure 7. Explain the application of Williamson-Hall equation.

elastic strain, which is about (0.0011). It is a small enough, that is good because it is a function to the stability of the cubic phase produced.

\section{Conclusion}

The synthesis ability a metal nanoparticle is valid experimentally during the reduction method. The size of nanoparticle is about $(20 \mathrm{~nm})$, which is favor than that imported from the external company. The output nanoparticle powder is suitable to apply in the medical field through the obtainable results.

\section{Acknowledgements}

This research is done due to the cooperation of Al-Nahrain University/College of Science with Kırşehir Ahi Evran University/Art and Science Faculty/Turkey. It is done at Al-Nahrain University/College of Science/Physics Department during the supervision on M.Sc. student

\section{Conflicts of Interest}

The authors declare no conflicts of interest regarding the publication of this paper.

\section{References}

[1] Lai, F., Lin, Z. and Zhang, P. (2018) Preparation and Characterization of Mi- 
cro/Nano-Silver Powders. AIP Conference Proceedings 1995, pp. 0200101-0200105.

[2] Turkevich, J., Stevenson, P.C. and Hillier, J. (1951) A Study of the Nucleation and Growth Processes in the Synthesis of Colloidal Gold. Discussions of the Faraday Society, 11, 55-75. https://doi.org/10.1039/df9511100055

[3] Zhao, P., Li, N. and Astruc, D. (2013) State of the Art in Gold Nanoparticle Synthesis. Coordination Chemistry Reviews, 257, 638-665. https://doi.org/10.1016/j.ccr.2012.09.002

[4] Ji, X., Song, X., Li, J., Bai, Y., Yang, W. and Peng, X. (2007) Size Control of Gold Nanocrystals in Citrate Reduction: The Third Role of Citrate. Journal of the American Chemical Society, 129, 13939-13948. https://doi.org/10.1021/ja074447k

[5] Zhang, H.X., Siegert, U., Liu, R. and Cai, W. (2009) Facile Fabrication of Ultrafine Copper Nanoparticles in Organic Solvent. Nanoscale Research Letters, 4, Article No. 705. https://doi.org/10.1007/s11671-009-9301-2

[6] Camacho-Flores, B.A., Martínez-Álvarez, O., Arenas-Arrocena, M.C., Garcia-Contreras, R., Argueta-Figueroa, L., de la Fuente-Hernández, J. and Acosta-Torres, L.S. (2015) Copper: Synthesis Techniques in Nanoscale and Powerful Application as an Antimicrobial Agent. Journal of Nanomaterials, 2015, Article ID: 415238.

https://doi.org/10.1155/2015/415238

[7] Nouailhat, A. (2006) An Introduction to Nanoscience and Nanotechnology. Wiley, New Jersey.

[8] Wang, H., Qiao, X., Chen, J. and Ding, S. (2005) Preparation of Silver Nanoparticles by Chemical Reduction Method. Colloids and Surfaces A: Physicochemical and Engineering Aspects, 256, 111-115. https://doi.org/10.1016/j.colsurfa.2004.12.058

[9] Kanwal, Z., Raza, M.A., Riaz, S., Manzoor, S., Tayyeb, A., Sajid, I. and Naseem, S. (2019) Synthesis and Characterization of Silver Nanoparticle-Decorated Cobalt Nanocomposites (Co@AgNPs) and Their Density-Dependent Antibacterial Activity. Royal Society Open Science, 6, 1821351. https://doi.org/10.1098/rsos.182135

[10] Mehta, B.K., Chhajlani, M. and Shrivastava, B.D. (2017) Green Synthesis of Silver Nanoparticles and Their Characterization by XRD. Journal of Physics: Conference Series, 836, 012050. https://doi.org/10.1088/1742-6596/836/1/012050 